\title{
The effectiveness of a study abroad program for increasing students' cultural competence
}

\author{
Helen C. Ballestas, Maureen C. Roller \\ School of nursing, Adelphi University, New York, USA \\ Correspondence: Helen Christina Ballestas. Address: School of nursing, Adelphi University, New York, USA. Email: \\ hballestas@adelphi.edu. \\ Received: October 10, 2012 \\ Accepted: November 29, 2012 \\ Online Published: January 7, 2013 \\ DOI : 10.5430/jnep.v3n6p125 \\ URL: http://dx.doi.org/10.5430/jnep.v3n6p125
}

\begin{abstract}
Background: Measuring the effect of a study abroad program is one way to determine its impact on developing students' cultural competence. To date, few articles describe outcomes of such strategies. In some published studies, a description of the program or intervention is frequently not offered making it difficult to utilize findings or to replicate them.

Purpose: To measure the effects of a study abroad program utilizing a structured course to improve the cultural competence of baccalaureate nursing students.

Methods: A comparative quantitative design measured level of cultural competency before and after a study abroad program.

Results: The results demonstrated an $89 \%$ improvement in cultural competence following this experience.

Conclusions: A short-term study abroad experiential learning program can increase cultural competence for baccalaureate nursing students. Quantitative measures of effectiveness and a description of the program's content and format are essential in determining a study's strength.
\end{abstract}

\section{Key words}

Cultural competence, Study abroad, Undergraduate education, Costa Rica

\section{Introduction}

Because today's nurses care for culturally diverse patients, nursing requires culturally competent practitioners. Faculty responsible for preparing nurses who will provide culturally relevant care need effective approaches for teaching transcultural nursing. Throughout the literature, educators of undergraduate students have described courses and experiences to assist students achieve this competence. Experiential learning has been well-recognized as a method for changing attitudes, an important dimension of cultural competence. Study abroad programs can provide students with this type of learning. Measuring the effectiveness of such a program is an essential part of ongoing curriculum evaluation. This article reports on the effects of a short term study abroad experience in Costa Rica for a group of undergraduate nursing students. The program's format was designed to increase cultural competence. The limited literature on effectiveness of such programs is explored. The study methods and results are presented and are discussed within the current published literature on this topic. Implications for education and recommendations for further study are provided. 


\subsection{Cultural competence: definitions, models and measurements}

In nursing, cultural competence is important for an understanding of the cultural and social influences of clients' health and related behaviors ${ }^{[1]}$. Cultural competency is an ongoing process; one never reaches the end and is continually learning. Campinha-Bacote views cultural competence as the process in which the health care professional continually strives to effectively work within the cultural context of a client (an individual, family or community) ${ }^{[2]}$. The definition of what it is to be culturally competent has developed over the years. Campinha-Bacote explains that it is not enough today to be aware, the nurse must "become" and be motivated to engage in and develop cultural awareness and competency ${ }^{[2]}$.

Models have emerged that guide and support the evolving definition of cultural competence and include CampinhaBacote's model: The Process of Cultural Competence in the Delivery of Healthcare Services as well as the LEARN model ${ }^{[2,3]}$. Within Campinha-Bacote's model, cultural awareness which encompasses sensitivity, cultural knowledge, cultural skill, cultural encounters and cultural desire interact to assist the learner envision an understanding of and competence with different cultures and their members ${ }^{[2]}$.

Rew et al's model used frequently in nursing includes the four dimensions of cultural competence: cultural awareness (affective dimension), cultural sensitivity (attitudinal dimension), cultural knowledge (cognitive dimension), and skills (behavioral dimension) ${ }^{[4]}$. These authors claim that when individuals are conscious that people are different from one another partly because of their cultural backgrounds, this is awareness. However when individuals value and respect these cultural differences, they are said to be culturaly sensitive ${ }^{[4]}$. Awareness and sensitivity overlap and in the CampinhaBacolt's model awareness encompasses sensitivity ${ }^{[2]}$.

Seminal work by Leininger on transcultural nursing was the catalyst for nursing programs to incorporate "cultural diversity" and cultural constructs into curricula ${ }^{[6]}$. Many nurse educators believed that discussing different cultures with students often highlighted important differences; unfortunately, in many instances, this resulted in reinforcing stereotypes. That is, individuals from a specific culture were discussed as homogenous. To date, nursing continues to refine both the importance of and the method for teaching cultural competence. Knowing that diversity exists and being "sensitive" to cultural differences have evolved today into the process of cultural competence.

In addition to models, several instruments have been developed that measure one of more of the dimensions considered a part of cultural competence. However, some of these measures may not be useful if educators are seeking a comprehensive measure of cultural competence. The more recent instruments, such as the Campinha-Bacote's tool, used in this study, measure all dimensions of cultural competence ${ }^{[7,8]}$. Educators should also be aware that some instruments developed are more appropriate for use when studying healthcare providers in general and some are specifically designed for nurses.

\subsection{Cultural competence and nursing education}

Numerous published reports have focused on educating students about culture through classroom instruction and experiential learning. Cultural awareness and sensitivity have been proposed as significant content for nursing curricula and practice in many countries. For example, teaching cultural awareness is evident in Papp's and Ramsden's work in New Zealand for which they discussed the importance cultural safety within a colonial context ${ }^{[9]}$. In 1992, the Nursing Council of New Zealand defined cultural safety as "The effective nursing of a person/family from another culture by a nurse who has undertaken a process of reflection on own (sic) cultural identity and recognizes the impact of the nurses' culture on own ( sic) nursing practice” ${ }^{[9]}$.

In Australia, Downing, Kowal and Paradies identified that culturally competent care was not being provided to the

indigenous population ${ }^{[10]}$. They believed that education regarding cultural competence takes on breadth and depth when cultural competency is learned through "servicing" the population directly that is, providing an experiential format for learning. Zoucha, Mayle and Colizza called for the blending of classroom constructs with service learning which would enrich the development of cultural awareness and eventual competence ${ }^{[11]}$. 
In the United States, some nurse educators teach students about cultural awareness and sensitivity by utilizing entire courses, and others have developed content that is threaded throughout a curriculum. Hughes and Hood developed and incorporated cultural constructs throughout an entire nursing program in order to develop cultural competence for undergraduate nursing students ${ }^{[4]}$. They used a variety of learning methods including didactic and experiential learning such as poster presentations, and required self-reflective journals-methods deemed important for cultural awareness. In order to demonstrate an increase in students' cultural sensitivity at the end of the program, they used a cross-cultural evaluation tool that measured behaviors and attitudes and administered it in one major course.

Experiential formats have also been described by nurse educators. Robinson, to increase cultural sensitivity, assigned students to interview a nurse from a minority group ${ }^{[12]}$. Following this, she established a panel presentation of minority nurses who were not the interviewees from the first assignment. Students were then required asked to describe their attitudes in a reflection paper. The author found that by utilizing the reflection papers for student evaluation, she could determine the students' growth regarding their cultural sensitivity.

To date, there has been little comparison of effective methods for teaching cultural competence. Thus, Kardong-Edgren et al. maintain there is no definitive approach to teaching strategies that enhance cultural competence ${ }^{[13]}$. One strategy for teaching and developing cultural competence in students that has received a significant amount of attention in the literature is the use of study abroad programs.

\subsection{Cultural competence and study abroad programs}

Study abroad programs are viewed as an important strategy because they provide hands on or experiential learning, considered important in changing attitudes regarding culture. This format is also valued because with overcrowded nursing curricula today, constructs and theories can be taught outside of the requirements in some content overloaded programs. However, despite their popularity, there is limited applicable research on study abroad programs and their effect on developing or improving cultural competence. The reported research also lacks information on measurements used to determine effectiveness. As well, many studies focus on only one or two dimensions of competence such as awareness or sensitivity. In addition, there is also a wide variety of study abroad programs that have been reported. One noteworthy variation in programs is the length of the experience thus making it difficult to translate and utilize findings from studies to develop curricula. Finally, many of the studies that report effectiveness in increasing cultural competence do not describe the educational experiences in which students have been engaged, preventing replication.

Because many of the reports on study abroad programs have anecdotal findings or are qualitative in design, educators have called for more evidenced based research on this topic. Although the reported qualitative studies are rigorous regarding the methodology used, a major drawback is that they do not provide measureable outcomes of their program's effectiveness [14-16]. Moreover, in evaluating the current research on study abroad programs, only short term outcomes, those immediately following the experience, have usually been reported. Further it is unknown in some of the reported research if the positive outcomes were maintained beyond the immediate end of the program ${ }^{[17]}$.

In reported quantitative studies, researchers have demonstrated findings such as positive attitude changes using, for example, the Cultural Awareness Scale. Students studied in Mexico at both a language school and a university in one specific reported study in which the program planned for four weeks in duration was and extended to six weeks ${ }^{[18]}$. For this study, the authors reported that quantitative data collected pre-experience was supported by the qualitative data the obtained. The post-experience questions addressed how the experience affected students' knowledge, attitudes and skills, and how the experience might influence a student's clinical practice. However, little description of the program and its strategies were presented in the report and it was therefore difficult to evaluate the findings.

Findings from two studies that used control groups as comparison to determine the effects of a study abroad experience demonstrated that students attending the study abroad program scored higher from pre-test to post test ${ }^{[19,20]}$. However, one study reported had an extremely uneven sample with 7 in the experimental group who had both classroom and study 
abroad experience, and 25 in the control group with only classroom experience. The uneven group numbers affected confidence in this study's quantitative findings ${ }^{[19]}$.

The second experimentally designed study used a larger sample. Researchers compared 80 senior students who had participated in a study abroad program with 120 who had not ${ }^{[20]}$. They administered the Cultural Self Efficacy Scale three times following the study abroad experience and found at these three data collection times statistically significant results between the two groups ${ }^{[20]}$. However, the experiences abroad and teaching strategies were not detailed in the report; another limitation was that although the students spent more than four months abroad, they lived in different cultures without a unified teacher- supervised educational strategy. The strength of this program was its length. To improve cultural competence, some authors recommend at least a 12 to 16 week program; however, the majority of the reported research on nursing students has been undertaken on 2-5 week programs ${ }^{[21,22]}$.

In a recently reported study, objectives, teaching strategies and the format for the study abroad program for nursing students were well described, thus making replication possible ${ }^{[23]}$. However, the effectiveness of the program was not based on an instrument to measure cultural competence but was evaluated by faculty on such objectives as how well students communicated, were flexible such as keeping an open mind, and were sensitive regarding respect of culture through interactions.

\subsection{Summary of literature and study's aim}

Following an integrative review of the literature on study abroad programs for nursing students, Edmonds concluded that, although study abroad has been called a useful strategy for bridging the theory-practice divide, the present body of nursing knowledge regarding the effect of study abroad programs on cultural competence is not sufficient to date to support this premise. In searching for evidence in the literature, we concluded that much of the published research on study abroad programs' effect on cultural competence could not support their effectiveness for the following reasons: studies failed to measure cultural competence and only measured one or two dimensions of the process; some studies used comparative designs but with vastly uneven samples; authors failed to present the objectives and strategies used for the programs; and lastly, there was a wide variation among reported investigations regarding the length of the programs implemented. We found that qualitative studies and anecdotal reports also determined effectiveness but these used student reports for data and a standardized measure on cultural competence was not used. Therefore, the aim of this study was to test the effectiveness of a short term, one week, study abroad program designed as a course to improve cultural competence for undergraduate nursing students.

\section{Methods}

\subsection{Design and framework}

The study design was a comparative quantitative one with a pre-test, post-test data collection period. The one week study abroad course served as the intervention that was tested.

The theoretical framework for this research study was Campinha-Bacote’s Cultural Competency Model ${ }^{[2,24]}$. The model highlights the four major dimensions of cultural competence presented earlier. Based on this model, the Inventory for Assessing the Process of Cultural Competency among Healthcare Professionals-Revised (IAPCC-R) ${ }^{[8]}$ was used with permission of the author.

\subsection{Ethical considerations}

Permission to conduct the research was obtained from the Institutional Review Board of the authors' university and received approval under the exempt status. The researchers provided participants with an information sheet about the 
study and a consent form to sign. Confidentiality was maintained by coding the questionnaires so that responses could not be linked to any individual student.

\subsection{Participants, setting and program description}

Purposive sampling of undergraduate baccalaureate nursing students was used. All students registered and attended the one credit elective course, On Site in Costa Rica. Subjects self-selected into the program and the research study. The sample size was 18. All 18 participants who enrolled in the program volunteered for the study. The subjects travelled to Costa Rica for this community service academic experience of approximately 6 days. The objectives and students' experiences on site for this course are in Table 1 and the related student required assignments appear in Table 2.

Table 1. Course Objectives and Experiences

\section{Objectives}

Upon completion of this course the student will be able to:

1. Discuss the interplay among culture, socio-economic issues and healthcare as observed during the student experience in Costa Rica.

2. Identify ways in which his/ her awareness of social responsibilities has increased.

3. Describe specific team members' skills that are important in the context of this global experience.

4. Describe the impact of this service-based experience on his/her personal and professional growth.

\section{Experiences in Country: Costa Rica}

1. Attend a two-hour class before the global experience.

2. Make clinical visits included a tour of the local main hospital in city center

3. Observe the native medicine man administering herbal medications and participate a tribal dance

4. Perform health assessments in a community program with 20 indigenous patients.

5. Set up and manage an outpatient clinic at a local school where vital signs, vision and dental exams are to be completed on over 200 children.

6. Participate in clinic experiences that include assessments, health teaching, glucose monitoring, breast exams, dressing care, mobility training and Hospice visits.

7. Become familiar with the cultural environs: students introduced to the delicate eco-system of Costa Rica. Visits to a local rain forest and a trip on a tropical river expose the students to the way of life of the indigenous people of Costa Rica.

8. Attend a post experience class and debriefing.

Table 2. Students' Assignments

\section{Pre experience class:*}

- Complete pre-experience cultural competence instrument.

- Attend a PowerPoint presentation by faculty on cultural competence.

- Complete reading assignments and participate in discussion on Costa Rica's culture, socio-economic conditions and health care;

- Students are evaluated on attendance, preparation for and participation in class.

\section{In-country:}

- $\quad$ Student attends all site experiences, completes a daily reflection journal, and participates in meetings on site.

- Daily activity for reflective journaling should be thoughtful, analytical, and not merely a summary of events.

- The journal should demonstrate critical and integrative thinking and demonstrate connections between topics and experiences.

\section{Post experience class:}

- $\quad$ Complete post-experience cultural competence instrument.

- $\quad$ Participate in debriefing concluding the experience.

- Compare and contrast in writing one's personal experiences in Costa Rica related to cultural competence to one or more peer reviewed journal articles on the topic. 


\subsection{Data collection}

Each participant's descriptive information was obtained using a Demographic Form for students' age, gender, race/ ethnicity, current college credits, past experiences with travel outside the United States, and to determine if participants spoke Spanish fluently. The Inventory for Assessing the Process of Cultural Competency among Healthcare ProfessionalsRevised (IAPCC-R) ${ }^{[8]}$ was distributed before and after the Costa Rica experience.

\subsection{I nstrument}

The Inventory for Assessing the Process of Cultural Competency among Healthcare Professionals-Revised (IAPCC-R) was used for the study ${ }^{[7,8]}$. This instrument was selected because it is a measure of all of the "dimensions" of cultural competence and is based on the framework used for this study. The IAPCC-R measures the cultural constructs of desire, awareness, knowledge, skill and encounters. This instrument has been widely used nationally and internationally. The instrument is a pencil and paper self-assessment tool with item responses on a 4-point Likert-type scale using strongly agree, agree, disagree, and strongly disagree; there are 25 items. The total possible scores up to 100 are designated as cultural proficiency from 91-100, culturally competent 75-90, culturally aware 51-74, and culturally incompetence $50-25^{[8,25]}$. When the total response score increases, this indicates a stronger agreement, or more proficiency in cultural competence; as the scores decrease, the responder is in disagreement with the cultural competency statements, and earns a lower overall competency score. Completing the instrument takes approximately 20 minutes and was administered before and after the Costa Rica experience.

Psychometrics of the instrument have been reported by the author ${ }^{[8]}$. To date, for reliability, Cronbach's Coefficient alphas have ranged from 0.70 to 0.90 across numerous studies. Internal validity has been confirmed through a number of studies ${ }^{[8]}$. Construct validity was established in a study comparing the IAPCC-R to the Ethnocentrism Scale ${ }^{[25]}$. Content and face validity were established by a panel of experts in transcultural healthcare ${ }^{[8]}$.

\section{Results}

\subsection{Demographic data}

The participants' ages ranged from 20-47. The mode was 21 years (38.9\%). Gender was predominately female (94.4 \%). Race/Ethnicity was self -reported as Black/African American $\mathrm{N}=3$; Asian, $\mathrm{N}=2$; White $\mathrm{N}=8$; Hispanic/Latino $\mathrm{N}=1$ and Other $N=4$. The students 'demographics in the school from which the sample was recruited was compared to the study's because all nursing students in the school were eligible to participate. The comparisons yielded the following: for gender, there was a lower percentage of males in the study than the school (School N=13.1\%); African American and Asian study participants were similar in percent to the school's population; the study participants were $44 \%$ white, whereas in the school, the student body is 55\% white. The study had $5.56 \%$ Hispanic/Latino students; this was lower than the school's $13.4 \%$. A higher percentage of the study's 18 participants (22\%) self-reported their race-ethnicity as "other" compared to only $3.4 \%$ of the school's population. Probably the most remarkable statistic was the lower percentage of participants that was Hispanic/Latino and noteworthy because the culture, the site for the program, is Hispanic. However, only two participants reported speaking Spanish fluently.

Course credits completed by the subjects at the time of the program ranged from 60-120. The mode was 90 credits, for $33.3 \%$ of the subjects. With respect to former travel (pleasure) outside of the US, $72 \%$ reported such experiences that included Central America 22.2\%, South America and the Caribbean 5.6\%, Asia and Europe 5.6\%,only Europe 5.6\%, the Caribbean and Europe 11.1\%, Asia 5.6\%, Asia and Caribbean 5.6\% and Caribbean 16.7\%.

\subsection{Effectiveness of experience on cultural competence}

With respect to the purpose of the study, which was to increase cultural competence using a study abroad program as the intervention, scores were compared for pre and post questions (items 1-25) on the IAPCC-R for the18 subjects. Scores 
increased on 16 (88.8\%) of the 18 students. The overall mean score pre- experience was 66.3 of 100 possible. The overall mean score post- experience increased to 78.8. A $t$-test used to compare the means of independent samples (pre and post) demonstrated significance $(t=5.62 ; p<.000)$.

Scores on the completed instrument corresponded to the four levels determined by Campinha-Bacote ${ }^{[8]}$ as follows: for the pre experience: 0 subjects scored in the culturally incompetent level; 17 (94.4\%) subjects were culturally aware; 1 subject (5.6\%) was culturally competent and 0 subjects scored culturally proficient. For the post experience, the scores were recorded as 0 subjects scored in the culturally incompetent level, 4 (22.3\%) subjects were culturally aware, 14 (77.7\%) subjects were culturally competent and 0 subjects scored culturally proficient. The most significant change was for the culturally competent scale was for 13 students in the culturally aware category at the pre-test who increased their scores to the culturally competent level at post-test.

\subsection{Study limitations}

This study had a small sample size of 18 subjects. The study was conducted in one location with one class of undergraduate baccalaureate nursing students attending one university and therefore limits generalizability. The study did not include a follow up post-test beyond the immediate post experience, one that would have determined if effects were maintained beyond the program's end. The instrument was validated and reliable. However, a student version (SV) of this instrument has been recently published and this may have been more appropriate to use ${ }^{[26]}$.

The students in this study were mostly juniors in a four year program and it unknown how much prior content on cultural awareness, sensitivity and competence, threaded throughout their curriculum, and might have affected the results. The larger percent of Hispanic students in the sample compared to the school's population may have reflected a specific interest for these students in a site that was also a Hispanic culture.

\section{Discussion}

This study tested the effectiveness of a short term study abroad program on undergraduate nursing students' increase in cultural competence. The study findings supported the Capinha-Bacote's model as a framework for, and the IAPCR instrument as a measure of, cultural competence ${ }^{[2,8,25]}$. Using an instrument that measures all dimensions of competence assists faculty to determine more than knowledge acquisition, cultural sensitivity or awareness, because it focuses on the higher or more complex learning of application or behaviors. Using an instrument developed for health professionals, especially because the content of the study abroad program was health-related, was another important consideration regarding this framework and instrument selection.

Because of the differences reported in studies on the types of study abroad programs especially their length, it is difficult to compare this study's findings to findings from other quantitative investigations. As presented earlier, types of programs reported in the research literature were not well described regarding their goals, objectives and experiences for students which also prevented a comparison with our findings. However, similar to other quantitative studies that demonstrated an increase in some aspect of cultural competence, this program was effective as a short study abroad program.

The present program was only one week in duration. When comparing programs' effectiveness, educators need to consider and differentiate terms such as study abroad, exchange programs or immersion programs. No standard definitions exist for length of time or format for these different types of programs. Authors of most of these reported investigations concluded that the longer the program in duration, the more significant and lasting the benefits with respect to cultural sensitivity. For example, Zorn demonstrated a more significant effect using two groups of students for those students who participated in the longer experience 12-16 weeks vs. 3-4 weeks ${ }^{[22]}$. However, students had very different experiences during the longer period experiment; they were also "immersed" in many different countries. DeDee and Stewart claimed that a time frame of 12-16 weeks was the ideal length for study abroad ${ }^{[21]}$. Conversely, St. Clair and McKenry demonstrated that two groups, short and long term program participants, did not differ on an increase in Cultural Self Efficacy at the end of their 
programs ${ }^{[20]}$. However, at a later time, at graduation, the seniors who participated in the longer program maintained their cultural self-efficacy scores while the shorter group participants' scores decreased.

The present study adds to the body of knowledge on the effectiveness of study abroad programs on cultural competence. Unlike some of the reported studies, this investigation was quantitative in design and used a valid and reliable measure that considered all of the dimensions of cultural competence, pre and post experience. The program's objectives and assignments were outlined to allow for replication.

The choice of a long vs short immersion is for most college nursing programs primarily based on students' course work schedules and financial resources. Many studies in the literature reported findings for longer term study abroad programs but none of these enrolled nursing students. However, given the constraints of undergraduate nursing students, a short term program such as this can be effective.

The present study design would be enhanced if reinforcement of learning for those who participated in the study abroad program or for those classmates in the school who could not participate would be added. How applicable the leaning abroad might be could also be determined by measuring how students on their return from such a program actually relate to diverse populations in their practice.

\section{Implications and recommendations}

Undergraduate nursing students exposed to a different culture of clients than in their home environment can gain some proficiency in cultural competence through a study abroad program. Even short programs can have the benefit of additional learning beyond what students glean from their traditional classroom course work. Faculty members should determine ways to ensure that students' achievements in cultural competence following such programs are maintained; therefore the measurement of effectiveness over a longer period of time is essential. Influencing other students' on competence who have not had the opportunity to study abroad might also is a strategy; this would also continue to reinforce the participants' learning.

While this study did not use qualitative data as a measure of effectiveness, such data, especially regarding students' evaluation of the program and the course work, can further enhance and improve a program and should be considered . This type of information may influence outcomes of student learning and acquisition of proficiency. Using the same country for annual experiences has the benefit of providing the faculty an opportunity to continually refine their program and the important relationships in the host country.

In order to use reported research findings, nurse educators must continue to be aware of the differences in the definitions of terms such as cultural awareness, sensitivity and competence used in investigations. Clarification is also needed in the literature regarding whether a program is a study abroad program, an exchange program, or an immersion program. Furthermore, in order to promote replication, researchers should include in their reports the program's goals, objectives and strategies on which research on effectiveness is based.

\section{Conclusion}

Short-term, financially feasible study abroad programs can produce changes in cultural competence for nursing undergraduate students. This study's results add to the literature on the effectiveness of such a program. The study also provided support for the specific Campinha-Bacote model and its relevant instrument on cultural competence for health professionals ${ }^{[2,8,24]}$. A description of the program that was included may assist other nurse educators in planning such an experience. The students' acquired cultural proficiency beyond the post-test phase needs to be considered through a second data collection period perhaps a year later. As well, comparisons of students who participate in such an experience 
and those who have had only the traditional learning for cultural content and competence should be undertaken. Further studies might also address the application of increased cultural competence on students' clinical practice.

\section{References}

[1] Betancourt R C, Green AR, Ananeth-Frempong II O. Defining cultural competence: A practical framework for addressing racial and ethnic disparities in health and health care. Public Health Reports. 2003; 118: 293-302 http://dx.doi.org/10.1093/phr/118.4.287

[2] Campinha-Bacote J. The process of cultural competence in the delivery of health care services: A model of care. Journal of Transculural Nursing. 2002; 13: 181-184. PMid:12113146 http://doi: dx.doi.org/10.1177/10459602013003003

[3] Berlin E, Fowkes W.A teaching framework for cross-cultural health care. Western Journal of Medicine. 1982; 139:934-938.

[4] Rew L, Becker H, Cookston J, Khosropour S, Martinez S. Measuring cultural awareness in nursing students. Journal of Nursing Education. 2003; 42: 249-257. PMid: 12814215 NLM UID: 7705432

[5] Hughes K \& Hood LJ. Teaching methods and an outcome tool for measuring cultural sensitivity in undergraduate nursing students. Journal of Transcultural Nursing. 2007; 18: 57-62. PMid:17202530 http://dx.doi.org/10.1177/1043659606294196

[6] Leininger M M. Transcultural nursing; concepts and practice. John Wiley: New York,1978

[7] Campinha-Bacote J. (2012a). Cultural Competency in Healthcare Delivery: Have I ASKED' Myself The Right Questions? Available from: http://www.transculturalcare.net/Cultural_Competence_Model.htm

[8] Campinha-Bacote J.).About the IAPCC-R [Internet]. Available from: http://www.transculturalcare.net/iapcc-r.htm

[9] Papps E, Ramsden, I. Cultural safety in nursing: the New Zealand Experience. International Journal of Quality in Health Care.1996; 8: 491-497. http://dx.doi.org/10.1016/S1353-4505(96)00080-4

[10] Downing R, Kowal E, Paradies A Y. Indigenous cultural training for health workers in Australia. InternationalJournal of Quality in Health Care. 2011; 23: 247-257. http://dx.doi.org/10.1093/intqhc/mzr008

[11] Zoucha R, Mayle K, Colizza D. The bridging of transcultural response In health care and service learning courses in a community based baccalaureate nursing curriculum: A natural connection. Online Journal of Cultural Competence in Nursing and Healthcare (Internet). 2011; 1. Available from: http://www.cultural-competence-project.org/ojccnh/1/4/1-10.pdf

[12] Robinson, J H Increasing students’ cultural sensitivity: A step toward greater diversity in nursing. Nurse Educator. 2000; 25:131-135. htpp://dx.doi.org/223-200005000-0001310.1097/00006c

[13] Kardong-Edgren S, Cason C, Brennan A, Reifsnider E, Hummel F, Mancini M, Griffin C. Cultural Competency of Graduating BSN Nursing Students. Nursing Education Perspectives. 2010; 31: 278-285 http://dx.doi.org/ 10.1043/1536-5026-31.5.278.

[14] Ruddick HC, Turner D S. Developing cultural sensitivity: Nursing students' experiences of a study abroad program. Journal of Advanced Nursing. 2007; 59: 361-369. http://do.org/ 10.1111/j.1365-2648.2007.04312.x

[15] Edmonds M L. The lived experience of nursing students who study abroad: A qualitative inquiry. Journal of Studies in International Education. 2010; 14: 545-568. http://dx.doi.org/10.1177/1028315310375306

[16] Mkansawire -Valhmu L, Doering J. Study abroad as a tool for promoting cultural safety. Journal of Transcultural Nursing. 2012 ; 23 : 82-89. http://dx.doi.org/10.1177/1043659611423831

[17] Edmonds ML. An integrative literature review of a study abroad program for nursing students. Nursing Education Perspectives. 2012; 33: 30-34. http://dx.doi.org/10.5480/1536-5026-33.1.30

[18] Carpenter LJ, Garcia AA. Assessing outcomes of a study abroad course for nursing students. Nursing Education Perspectives. 2012; 33: 85-89. http://.dx.doi.org/10.5480/1536-5026-33.2.85

[19] Caffrey R, Neander W, Markle, D, Stewart B. improving the cultural competence of nursing students: Results of integrating cultural content in the curriculum and an international immersion. Journal of Nursing Education. 2005; 44: 234-241. PMid: 12814215 NLM UID: 7705432

[20] St Clair A, McKenry L. Preparing culturally competent practitioners, Journal of Nursing Education. 1999; 38:228-235. PMid: 10438097 NLM UID: 7705432.

[21] DeDee LS, Stewart S. The effect of student participation in international study. Journal of Professional Nursing. 2003 ; 19 (4): $237-242$. http://dx.doi.org/10.1016/S8755-7223(03)00086-3

[22] Zorn C R.Thelong term impact on nursing students participating in international education. Journal of Professional Nursing.1996; 12: 266-272. http://dx.doi.org/10.1016/S8755-7223(96)80056-1

[23] Foranda C, Belknap R A. Transformative learning through study abroad in Low income countries. Nurse Educator. 2012 ; 37 : 157-161. http://dx.doi.org/1097/NNEOB013e31825a879d

[24] Campinha-Bacote J. The process of cultural competency in the delivery of healthcare services: A culturally competent model of care [Internet]. 2003. Available from: http://www.transculturalcare.net/Cultural_Competence_Model.ht

[25] CapellJ, Dean E, Venstra G. The relationship between cultural competence and ethnocentrism of healthcare professionals. Journal of TransculturalNursing, 2008: 19: 120-125. http://.dx.doi.org/10.1177/1043659607312970

[26] Campinha-Bacote J. The IAPCC-SV (student version. Available at Transcultural Care Associates [Internet]. Available from: http://www.transculturalcare.net/iapcc-r.htm 